\section{A Possible New Cure for Acarine Disease of Honeybees}

Acarine disease, sometimes still known as 'Isle of Wight disease', as its name implies is caused by the invasion by the mite Acarapis woodi of the thoracic tracheæ of the adult honeybee. Although no longer epidemic, this complaint still causes the complete destruction of many colonies of bees annually and the serious weakening of many others.

To kill the mites inside the tracheæ of an infected bee, some volatile substance which exerts a marked differential toxicity upon the bee and $A$. woor $i$ would appear necessary. Up to the present a, small number of substances or mixtures has been found to have properties tending towards the desired objective. These have not, however, been found to be entirely satisfactory. Work has therefore been carried out here during the last two and a half years in an attempt to find a volatile material which kills $A$. woodi in the tracher of the honeybee, does not harm the adult bees or their brood, and is simple and cheap to apply. Many substances were tested with varying degrees of success and it has been found that the vapour of terpineol, a heavy faction of crude pine oil, gives the results desired, being highly toxic to $A$. wood $i$ but harmless to bees. In one experiment twenty-five bees which were heavily infected were subjected to the vapour of terpineol for five days and, at the end of this period, all the mites were found to be dead, whereas twenty control bees were all found to contain many live mites.

Experiments are now being conducted to determine a simple and satisfactory method of administering terpineol to a colony of bees.

It is hoped that the results obtained with this substance will be published in full in the near future.

Bee Department, C. G. Burrler.

Rothamsted Experimental Station, Harpenden. June 20.

\section{Production of Electron Pairs}

Some time ago we described a $\operatorname{method}^{1}$ for calculating rigorously (neglecting screening) the crosssection for production of electron-positron pairs in the field of a nucleus by a beam of $\gamma$-rays. This method also gave the energy distribution of the electrons or positrons. Resủlts were given for lead, atomic number $Z=82$, for $\gamma$-rays of energies $h \nu=5 m c^{2}$ and $3 m c^{2}$, and were in reasonably good agreement with experiments on pairs produced in

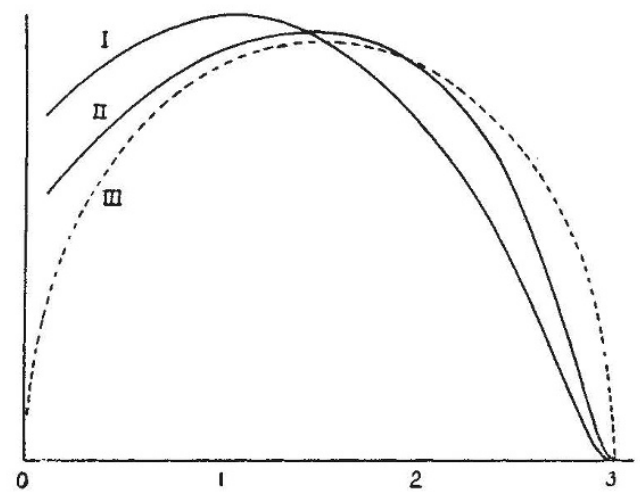

thin foils. Calculations have now been made for $Z=54, h \nu=5 m c^{2}$, to enable direct comparison to be made with Wilson chamber experiments on xenon and iodine. The final results contain about 20 per cent of (additive) extrapolated terms and should be accurate to about 10 per cent. The old calculation for lead has been extended and a small error corrected : the revised figures are given below.

The values of $\sigma$, the cross-section per atom for absorption by pair production by $\gamma$-rays of energy $5 m c^{2}$, for atomic numbers 82 and 54 are given in the table, together with the values calculated on the Born approximation ${ }^{2}$. The values of $\bar{E}_{+}-\bar{E}_{-}$are also given, where $\bar{E}_{+}$and $\bar{E}_{-}$. are the average energies of the positron and electron respectively.

\begin{tabular}{lll}
\multicolumn{1}{c}{$Z$} & 54 & 82 \\
$\sigma \times 10^{24}$ & $1 \cdot 1$ & $3 \cdot 0$ \\
$\sigma \times 10^{24}$ (Born) & $1 \cdot 1$ & $2 \cdot 5$ \\
$\left(\bar{E}_{4}-\bar{E}_{-}\right) / m c^{2}$ & $0 \cdot 27$ & $0 \cdot 51$
\end{tabular}

The energy distribution of the pairs produced by radiation of energy $5 \mathrm{mc}^{2}$ is shown in the accompanying figure. The abscissæ are electron-energies in units of $m c^{2}$ and the scale of the ordinates is arbitrary, the curves being adjusted to have the same area. Curves I, II and III are respectively for $Z=82, Z=54$ and the Born approximation. Curves I and II theoretically tend to a finite limit for zero energy of the electron, but these values have not been calculated.

University of Tasmania, J. C. JAEGER.

Hobart.

May 28.

${ }^{1}$ Jaeger and Hulme, Proc. Roy. Soc., A, 153, 443 (1936). See also NATURE, 13\%, 781 (1936).

${ }^{2}$ Bethe and Heitler, Proc. Roy. Soc., A, 146, 83 (1934).

\section{Thin Glass for Microscope Cover-Slips}

Mrcroscopists will note with interest and appreciation the recently recorded centenary of the production by Messrs. Chance Brothers of thin glass for microscopic purposes ${ }^{1}$.

There is good reason to believe that the production of this glass was due to the insistent and increasing demand by the early members of the Royal Microscopical Society. It is recorded that one of the first things the Council did in 1840 when thin flatted sheets first became available was to consider and standardize the size of the specimen slide at 3 in. $\times 1$ in.which size has since become universally adoptedand to install a device for the use of members for cutting both slides and cover-glasses from these sheets.

While it would be difficult to produce with certainty the earliest instance of the use of glass in place of talc, there is strong evidence to show that it was used for some ten years before thin flatted sheets became available through the enterprise of Messrs. Chance Brothers.

In 1831 Andrew Ross made the first achromatic objectives on Lister's formula. In $1832 \mathrm{~J}$. T. Cooper suggested the use of Canada balsam as a mounting medium for the preservation of microscopic specimens. Now in both these matters the use of the tale disk would appear to be impracticable ; and further, the development and purpose of the correction collar to high-power objectives which Ross brought out in 1837 was to correct the spherical and chromatic 